\title{
APRENDIZAGEM BASEADA EM PROBLEMA: UMA METODOLOGIA DE ANÁLISE DA VIABILIDADE ECONÔMICA PARA INSTALAÇÃO DE UM SISTEMA FOTOVOLTAICO
}

Laura Lisiane Callaidos Santos - laura.callai.santos@gmail.com Universidade Federal de Santa Maria - Campus Cachoeira do Sul Rodovia Taufik Germano, 3013 - Passo D'Areia 96503-205 - Cachoeira do Sul - Rio Grande do Sul

Jaderson Rosa dos Santos-r.jaderson@gmail.com Universidade Federal de Santa Maria - Campus Cachoeira do Sul Rodovia Taufik Germano, 3013 - Passo D'Areia 96503-205 - Cachoeira do Sul-Rio Grande do Sul

Leonardoda Silveira-leonardo_dasilveira@outlook Universidade Federal de Santa Maria - Campus Cachoeira do Sul Rodovia Taufik Germano, 3013 - Passo D'Areia 96503-205 - Cachoeira do Sul - Rio Grande do Sul

Cristiane Cauduro Gastaldini-crisgastaldini@gmail.com Universidade Federal de Santa Maria-Campus Cachoeira do Sul Rodovia Taufik Germano, 3013 - Passo D'Areia 96503-205 - Cachoeira do Sul - Rio Grande do Sul

Paulo CésarVargasLuz-paulo.c.luz@ufsm.br Universidade Federal de Santa Maria - Campus Cachoeira do Sul Rodovia Taufik Germano, 3013 - Passo D'Areia 96503-205 - Cachoeira do Sul - Rio Grande do Sul

Resumo: O processo de Aprendizagem Baseada em Problemas (PBL) é um método de aprendizagem ativa, onde os estudantes aprendem determinados assuntos no contexto de problemas reais. $O$ presente artigo demonstra a utilização do PBL no curso de Engenharia Elétrica da Universidade Federal de Santa Maria campus Cachoeira do Sul. Desse modo, é proposto aos alunos a análise da viabilidade econômica de um sistema fotovoltaico para um consumidor industrial do grupo A. Para a resolução do problema utiliza-se uma metodologia de dimensionamento, orçamento e análise econômica para a instalação de um sistema fotovoltaico. A metodologia foi desenvolvida em uma planilha em Excel para se automatizar os cálculos, o que torna o modelo facilmente aplicável a outros estudos de caso. Como principais resultados, obteve-se uma aprendizagem ativa dos conteúdos utilizados para a resolução do problema, aperfeiçoando o trabalho em equipe, as habilidades técnicas, comunicação, liderança e, assim, contribuindo na formação profissional completa e autônoma desses alunos.

Palavras-chave: Aprendizagem Ativa. Aprendizagem Baseada em Problemas. Sistema Fotovoltaico. Viabilidade Econômica. 


\section{INTRODUÇÃO}

As novas Diretrizes Curriculares Nacionais do Curso de Graduação em Engenharia estabelecem que deve ser estimulado o uso de metodologias para aprendizagem ativa, como forma de promover uma educação mais centrada no aluno (BRASIL, 2019). A aprendizagem ativa pode ser compreendida como o processo por meio do qual o estudante deixa de ser audiência para ser o protagonista no processo de aprendizagem. Assim, deixa de ser um receptor de informações, engajando-se de maneira ativa na aprendizagem e no desenvolvimento de habilidades, visando a construção do conhecimento (ELMÔR FILHO, 2019).

Dentre as técnicas de metodologias ativas, a Aprendizagem Baseada em Projetos (PBL) vem sendo amplamente adotada e estudada. A PBL é um método que transmite conhecimento de aprendizagem ativa, esse método leva os estudantes a aprender sobre os assuntos em um contexto de problemas reais e complexos (ELMÔR FILHO, 2019). Dentro desse contexto, em busca de aplicar os novos processos de ensino-aprendizagem, o Curso de Engenharia Elétrica da UFSM - Campus Cachoeira do Sul busca constantemente incentivar seus discentes a aplicar os conteúdos abordados durante a trajetória acadêmica em prol da comunidade.

Nesse sentido, verificou-se que há uma demanda local por estudos sobre a instalação de sistemas fotovoltaicos $(\mathrm{FV})$, principalmente quanto à viabilidade econômica dos mesmos. Isso ocorre devido a homologação de Resoluções Normativas (RN) que tratam sobre a inserção de Geração Distribuída (GD) pelos consumidores. A GD é uma forma de geração para consumo próprio, que consiste na geração de energia conectada ao sistema de distribuição de energia elétrica e que fica próxima aos centros de carga.

Nesse contexto foi proposto aos alunos do curso, o desenvolvimento de uma metodologia para projeto e a análise de viabilidade econômica de um sistema de geração de energia solar fotovoltaica. A metodologia é aplicada ao estudo de caso de uma indústria de Cachoeira do Sul, Unidade Consumidora (UC) do subgrupo A4 com tarifa verde, que planeja instalar o sistema. Os resultados foram obtidos por meio de uma planilha desenvolvida pelos autores no software Excel, com uma estrutura que permite que outros estudos de caso sejam realizados.

\section{PROJETO DO SISTEMA FOTOVOLTAICO}

No projeto de um sistema FV conectado à rede (grid-tie), adequado as necessidades do consumidor, será explorado o sistema de compensação de energia, instituído pela Resolução Normativa ANEEL no 482/2012 e alterado pela RN no 687/2015. Nesse sistema, a energia gerada e não consumida pela instalação é convertida em créditos pela distribuidora. Esses créditos poderão ser utilizados quando a energia demandada for maior do que a gerada, em um prazo máximo de 60 meses. Dessa forma, o dimensionamento da potência do sistema FV será baseado em uma média do consumo da UC nos últimos 12 meses (PINHO AND GALDINO, 2014).

Nesta seção, é descrito o método de dimensionamento do gerador FV e do inversor, principais componentes do sistema.

\subsection{Levantamento do histórico do consumo}

$\mathrm{Na}$ etapa inicial do projeto do sistema FV, é feito o levantamento do histórico de consumo, a partir das faturas de energia elétrica, com a finalidade de se determinar o consumo diário médio $(E)$ da instalação. Consumidores do grupo $\mathrm{A}$, alimentados em tensão maior ou igual a $2,3 \mathrm{kV}$, bem como consumidores do grupo B com tarifa branca, possuem uma diferenciação na tarifa de energia quanto ao horário de consumo. Nos horários do dia em que a demanda por 
energia elétrica é maior, o consumo é faturado à parte (consumo em ponta), com um valor maior ao que é praticado nas demais horas (consumo em fora de ponta).

Da mesma forma que o consumo possui tarifas distintas, a energia gerada em horários de ponta é convertida em mais créditos do que aquela gerada em horário de fora de ponta. Entretanto, como os horários de ponta encontram-se no período noturno, momento em que a geração FV é praticamente nula, considera-se que toda a geração ocorre em horários fora de ponta.

Por sua vez, o consumo dos créditos de energia nos horários de ponta também é maior. A razão entre as tarifas de energia em ponta e fora de ponta determina o fator de ajuste, Equação (1). Esse fator indica, na prática, quantas vezes o montante de energia a ser gerado em fora de ponta deve ser maior do que a demanda em ponta para supri-la (ANEEL, 2016).

Onde:

$$
F A=\frac{T E_{P}}{T E_{F P}}
$$

$F A$ : Fator de ajuste;

$T E_{P}(\mathrm{R} \$)$ : Tarifa de energia de ponta;

$T E_{F P}(\mathrm{R} \$)$ : Tarifa de energia de fora de ponta.

O consumo diário médio é calculado separadamente, para os horários de ponta e fora de ponta. O consumo diário médio em ponta, Equação (2) é dado pela soma do montante da energia faturada em ponta, nos últimos doze meses, dividido pelo total de dias faturados no ano.

Onde:

$$
E_{P}=\frac{1}{12} \cdot \sum_{i=1}^{12} \frac{C_{P, i}}{n_{i}}
$$

$E_{P}(k W h):$ Consumo diário médio em ponta;

$C_{P, i}(\mathrm{kWh})$ : Consumo faturado no mês $i$ em ponta;

$n_{i}$ (dias): Número de dias faturados no mês $i$;

De forma análoga, é calculado o consumo diário médio em fora de ponta, Equação (3).

Onde:

$$
E_{F P}=\frac{1}{12} \cdot \sum_{i=1}^{12} \frac{C_{F P, i}}{n_{i}}
$$

$E_{F P}(k W h):$ Consumo diário médio em fora ponta;

$C_{F P, i}(\mathrm{kWh})$ : Consumo faturado no mês $i$ em fora de ponta;

A próxima etapa do projeto do sistema FV consiste na avaliação do recurso solar.

\subsection{Avaliação do recurso solar}

$\mathrm{Na}$ avaliação do recurso solar, são observados a localização e o posicionamento dos módulos. Em suma, os aspectos considerados são: a inclinação e a orientação dos painéis, e a localização geográfica da instalação. A partir dessas informações, são estimados o valor da irradiação solar total diária média (ITD) e, por sua vez, o número de horas de Sol pleno (HSP). A ITD é igual ao montante de energia luminosa incidente por unidade de área, em um dia. O 
índice $H S P$, Equação (4) representa o tempo, em horas por dia, necessário para se obter a ITD a uma irradiância solar padrão de $1 \mathrm{~kW} / \mathrm{m}^{2}$ (PINHO AND GALDINO, 2014). Na próxima etapa do projeto do sistema é realizado o dimensionamento do gerador FV.

Onde:

$$
H S P=\frac{I T D\left(k W h / m^{2}\right)}{1 k W / m^{2}}
$$

$H S P(h)$ : Média diária das horas de Sol pleno;

ITD $\left(k W h / m^{2}\right)$ : Consumo faturado no mês $i$ em fora de ponta;

\subsection{Dimensionamento do gerador FV}

A potência do gerador FV, Equação (5), será dimensionada com base no consumo diário médio nos horários de ponta e fora de ponta e no número de horas de Sol pleno. Devem ser considerados, ainda, o fator de ajuste e uma taxa de desempenho (TD). A TD é um parâmetro utilizado para avaliar a potência real do sistema FV em face as diversas perdas envolvidas, tais como as perdas por sombreamento, por acúmulo de sujeira nos painéis e por queda de tensão nos condutores (PINHO AND GALDINO, 2014).

Os parâmetros $\alpha$ e $\beta$ serão utilizados para determinar, respectivamente, a fração da energia consumida em fora de ponta e em ponta que se deseja compensar. Ou seja, para $\alpha=\beta=1$, a potência de projeto será tal que o gerador será capaz de fornecer exatamente a potência média anual demandada pela UC.

Onde:

$$
P_{\text {gerador }}=\frac{\alpha \cdot E_{F P}+\beta \cdot E_{P} \cdot F A}{T D \cdot H S P}
$$

$P_{\text {gerador }}(k W h)$ : Potência do pico do gerador FV;

$T D$ : Taxa de desempenho do sistema;

$\alpha$ : Fator de compensação da energia em fora de ponta;

$\beta$ : Fator de compensação da energia em ponta.

O número total de módulos necessários para a geração da potência de projeto é dado pela Equação (6). Considera-se que todos os painéis possuem a mesma potência de pico.

Onde:

$$
n_{\text {modulos }}=\frac{P_{\text {gerador }}}{P_{\text {modulo }}}
$$

$n_{\text {modulos }}$ : Quantidade de módulos necessária;

$P_{\text {modulo }}(\mathrm{kWp})$ : Potência de pico de um módulo FV;

\subsection{Dimensionamento do inversor}

No dimensionamento do inversor, deseja-se que este opere o mais próximo da sua potência nominal, no maior tempo possível, de forma a não subutilizar o equipamento. A potência de pico do gerador $\left(P_{\text {gerador }}\right)$ nem sempre é fornecida pelos painéis $\mathrm{FV}$.

Com a finalidade de se compensar a subtilização do inversor, utiliza-se o fator de dimensionamento do inversor $(F D I)$. Esse parâmetro é utilizado para se determinar a potência nominal do inversor $\left(P_{\text {inversor }}\right)$ a partir a potência de pico do gerador e um valor arbitrado para FDI. 
A Equação (7) expressa o $F D I$, dado pela razão entre $P_{\text {inversor }}$ e $P_{\text {gerador }}$ (Pinho and Galdino, 2014).

Onde:

$$
F D I=\frac{P_{\text {inversor }}}{P_{\text {gerador }}}
$$

FDI: Fator de dimensionamento do inversor;

$P_{\text {inversor }}(\mathrm{kW})$ : Potência nominal do inversor;

$P_{\text {gerador }}(\mathrm{kWp})$ : Potência de pico do gerador FV;

\section{ANÁLISE ECONÔMICA}

Para validar economicamente um projeto, devem ser avaliados fatores como investimento, custos, retorno, dentre outros. Dentre as ferramentas empregadas na análise econômica, estão: fluxo de caixa, valor presente líquido, taxa interna de retorno e o Payback (ROSS ET AL., 2013).

\subsection{Fluxo de caixa}

O fluxo de caixa (FC) representa a soma da entrada e saída de capital durante um determinado período de tempo. O FC pode ser aplicado tanto para movimentações passados quanto futuras, em vista disso, o FC é uma ferramenta amplamente empregada em outros indicadores financeiros.

\subsection{Valor presente líquido}

O Valor Presente Líquido (VPL) é um dos indicadores financeiros mais utilizados para análise de investimentos. De acordo com Ross et al. (2013), VPL é o retorno financeiro gerado, descontado a uma taxa de juros, por um investimento futuro. Para calcular o VPL é necessário construir o fluxo de caixa do investimento e determinar a taxa mínima de atratividade (TMA) ou taxa de desconto. A Equação (8) apresenta o cálculo do VPL.

$$
V P L=\sum_{n=0}^{N} \frac{F C_{n}}{(1+i)^{n}}
$$

Onde:

$F C$ : Fluxo de caixa no período n;

$i$ : Taxa mínima de atratividade;

$N$ : Período total;

Se o VPL for maior que zero, significa que o investimento é viável, ou seja, há um retorno financeiro positivo. Caso o VPL seja menor que zero, o investimento em questão não é viável financeiramente. Quando o VPL for igual a zero, o investimento está em equilíbrio econômico, sendo assim, não há lucro ou prejuízo financeiro através do projeto.

\subsection{Taxa interna de retorno}

Para sintetizar os lucros de um investimento em uma taxa, utiliza-se a taxa interna de retorno (TIR). A TIR é obtida utilizando apenas os dados do próprio investimento em estudo, enquanto que a TMA é proveniente de informações conhecidas de outros investimentos. 
A TIR é a taxa na qual o investimento esteja em equilíbrio econômico quando utilizada como taxa de desconto. Em outros termos, a TIR é a taxa que torna o VPL igual a zero, sendo assim, é possível obtê-la através da Equação (8), substituindo a TMA pela TIR e igualando o VPL a zero (ROSS ET AL., 2013). Se a TIR for maior que a TMA, o investimento é viável economicamente quando comparado com um investimento que rende a TMA.

\subsection{Payback}

Payback (PB) é o período necessário para que o valor gerado pelo investimento seja equivalente ao valor do investimento. Apesar de ser um indicador amplamente utilizado, o PB, se comparado ao VPL, é um indicador menos sofisticado, pois o mesmo não leva em consideração os descontos durante o tempo. Sendo assim, o PB é empregado quando não há necessidade de uma análise detalhada (ROSS ET AL., 2013).

Após a revisão bibliográfica é apresentada a metodologia proposta no trabalho e o estudo de caso.

\section{METODOLOGIA}

A metodologia utilizada neste trabalho, objetiva o projeto de um sistema de geração fotovoltaico e a análise de sua viabilidade econômica, com base nos conceitos já apresentados. Todos os cálculos são realizados por meio de uma planilha construída no software Excel. A Figura 1 ilustra o passo a passo a ser seguido.

Figura 1 - Metodologia proposta.

Dimensionamento do Sistema FV e Análise Econômica

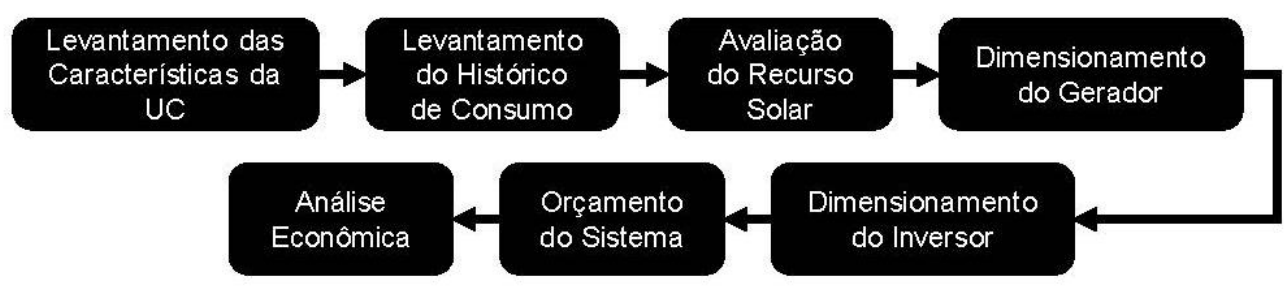

Fonte: Elaborado pelos Autores (2020).

Para início do projeto, é necessário conhecer as características gerais da instalação em que se deseja implementar o sistema FV, e as necessidades do consumidor. Nesta etapa, são definidos o local de instalação e a orientação dos painéis, atentando-se para aspectos estéticos e para a resistência mecânica das estruturas de fixação. Deve-se considerar, ainda, o limite máximo de geração de energia, dado pelas características da instalação e equipamentos utilizados.

Na sequência, são levantados os consumos mensais de energia da UC dos últimos 12 ou 24 meses, a partir das faturas de energia, para se obter os consumos diários médios em ponta $\left(E_{P}\right)$ e fora de ponta $\left(E_{F P}\right)$. Das faturas, são obtidos também os valores de tarifa de energia em ponta $\left(T E_{P}\right)$ e em fora de ponta $\left(T E_{F P}\right)$, para o cálculo do fator de ajuste $(F A)$ através da equação (1).

Na terceira etapa, da avaliação do recurso solar, é determinado o número de horas de Sol pleno (HSP) a partir da irradiação solar média diária incidente na localidade de instalação do sistema FV, através da Equação (4).

$\mathrm{Na}$ quarta etapa, dimensiona-se o gerador FV a partir das informações obtidas nos passos anteriores, como $E_{P}, E_{F P}, F A, H S P$. Os fatores $\alpha$ e $\beta$ são ajustados de acordo com as 
necessidades do consumidor e com as limitações da instalação. Determinam-se, então, a potência de pico do gerador $P_{\text {gerador }}$ e o número de painéis a serem instalados $n_{\text {modulos }}$ através das Equações (5) e (6).

A potência nominal do inversor é obtida ao se aplicar $P_{\text {gerador }}$ e um valor adequado de FDI na Equação (7), atentando-se para os limites de potência da instalação elétrica existente na UC. Este é o quinto e último passo do dimensionamento aqui proposto. Considera-se que o custo dos demais componentes do sistema FV, tais como cabos, conectores e estruturas é proporcional ao número de módulos a serem instalados.

Conhecidas as especificações do gerador e do inversor a sexta etapa, consiste em se realizar um orçamento do sistema. Com base em uma pesquisa, são estipulados os preços para os painéis, para o inversor, para as estruturas de fixação e para o projeto técnico. Esses valores determinarão o custo total do sistema.

A sétima e última etapa consiste na determinação do fluxo de caixa futuro, do valor presente líquido, da taxa interna de retorno e do payback. Esses indicadores serão utilizados para se concluir se o investimento é viável ou não.

\section{ESTUDO DE CASO}

\subsection{Levantamento das Características da UC}

O engenho é uma UC alimentada em $13,8 \mathrm{kV}$, um consumidor do subgrupo A4, com tarifa verde e, portanto, possui tarifações do consumo em ponta e em fora de ponta distintas. $\mathrm{O}$ rebaixamento da tensão é realizado por uma subestação no interior do engenho, que conta com um banco de transformadores que totaliza $1100 \mathrm{kVA}$ de potência.

O levantamento do histórico de consumo, foi realizado com base nas informações contidas nas últimas faturas de energia, fornecidas pelo engenho. Os dados de consumo, no período de setembro de 2018 a agosto de 2019, são apresentados na Figura 2.

Figura 2 - Gráfico do consumo diário médio por mês

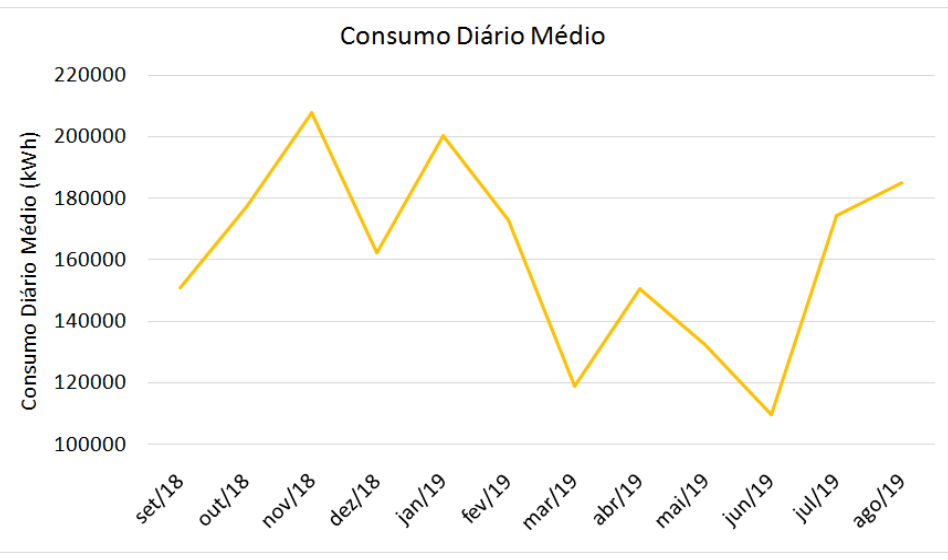

Fonte: Elaborado pelos Autores (2020).

A partir do histórico de consumo, foram obtidos os consumos diários médios em ponta $\left(\mathrm{E}_{\mathrm{P}}\right)$ e fora de ponta ( $\left.E_{F P}\right)$, conforme as Equações (2) e (3), e são, respectivamente, $E_{P}=259 \mathrm{kWh}$ e $\mathrm{E}_{\mathrm{FP}}=5.064 \mathrm{kWh}$.

As tarifas de uso do sistema de distribuição de ponta e de fora ponta são, respectivamente, $\mathrm{TUSD}_{\mathrm{P}}=\mathrm{R} \$ 1,0863677$ e TUSD $\mathrm{FP}=\mathrm{R} \$ 0,10803373$. As tarifas de energia de ponta e de fora ponta são, respectivamente, $T E_{P}=R \$ 0,56141135$ e $T_{F P}=R \$ 0,3369023$, conforme a 
concessionária de energia local. O fator de ajuste (FA) foi calculado através de (1), resultando em 1,666391958.

Os dados de irradiação para o local foram obtidos a partir da base de dados do programa SunData, disponível no site do Centro de Referência para Energia Solar e Eólica Sérgio Brito (CRESESB). A base tem a disposição informações de irradiação solar para diversas localidades, para quatro ângulos de inclinação conforme a Figura (3). Sabendo que a maior média anual é obtida com a inclinação de $22^{\circ} \mathrm{N}$, esta inclinação será adotada para os painéis.

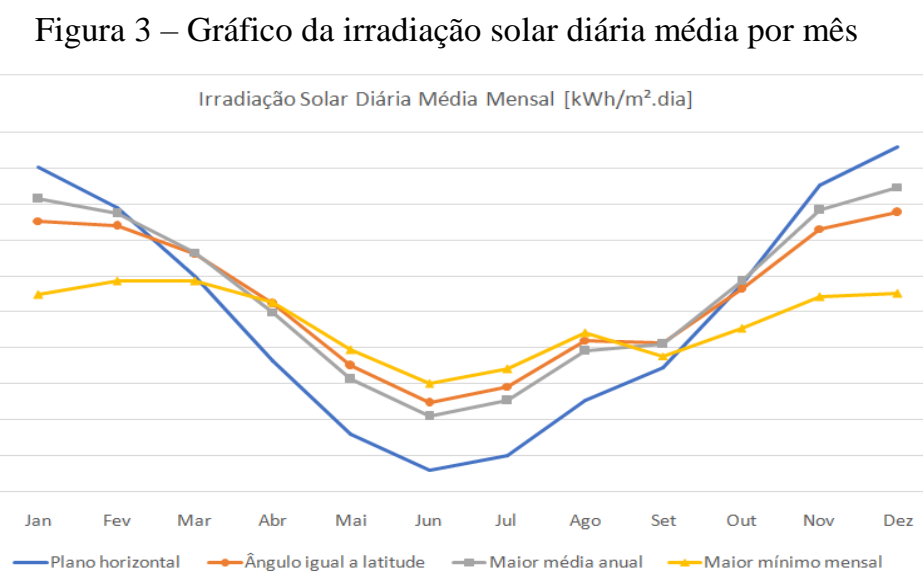

Fonte: Elaborado pelos Autores (2020).

Da figura 3, tem-se que o valor da irradiação solar total diária (ITD), para a inclinação de $22^{\circ} \mathrm{N}$, é igual a $4,73 \mathrm{kWh} / \mathrm{m}^{2}$. Ao se aplicar esse valor na Equação (4), tem-se que o número de horas de sol pleno (HSP) é dado por $4,73 \mathrm{~h}$. O próximo passo consiste em dimensionar o gerador.

\subsection{Dimensionamento do gerador e do inversor}

Para se determinar a potência de pico a ser gerada, utiliza-se a Equação (5). Foi arbitrada uma taxa de desempenho do sistema de $\mathrm{TD}=0,85$. Na Equação (5), o valor da potência de pico está em função dos fatores de compensação de energia em fora ponta e ponta, $\alpha$ e $\beta$. A Tabela (1) apresenta o valor de $\mathrm{P}_{\text {gerador }}$ para diferentes cenários, variando-se os parâmetros $\alpha$ e $\beta$. Para o cálculo da quantidade de painéis a serem utilizados, considerou-se uma potência individual de $330 \mathrm{Wp}$ por módulo.

Observa-se que, para gerar toda a energia demandada nos horários de ponta e fora de ponta, o gerador FV deve ter uma potência de pico de $4143 \mathrm{kWp}$, entretanto, esta potência é muito superior à potência do banco de transformadores existente na entrada da instalação, de 1100 kVA. Como este é um fator limitante à potência do sistema, o inversor deve possuir potência máxima de $1100 \mathrm{~kW}$. Utilizando a Equação (7) é possível determinar potência máxima do gerador, que é $1375 \mathrm{kWp}$ arbitrando-se um FDI de 0,8. Definidas as principais especificações do sistema FV projetado, a próxima etapa consiste em realizar a análise econômica do sistema.

\subsection{Análise econômica}

Nesta etapa, será realizada a análise econômica do projeto, para isso, é necessário definir os custos referentes aos componentes que integram o sistema FV. O painel fotovoltaico estabelecido neste trabalho foi o modelo Canadian CSI CS6U-330P, da marca Canadian Solar, com potência nominal de $330 \mathrm{Wp}$ e custo de $\mathrm{R} \$ 719,00$ (NEOSOLAR, 2020).

Optou-se por utilizar o inversor P55000Dc08A Pvs-100-Tl-Sx2-Full, da marca Abb, com potência de $100 \mathrm{~kW}$ e custo de $\mathrm{R} \$ 70.735,92$ (WINNERSHOP, 2020). 
A estrutura de fixação escolhida foi o suporte de fixação RSO-232C, onde é possível fixar até 4 painéis solares no solo. O custo do kit é $\mathrm{R} \$$ 836,07 (MINHACASASOLAR, 2020). Para o projeto, foi estabelecido um valor fixo de $\mathrm{R} \$ 40.000,00$.

Tendo em posse os valores dos componentes do sistema é possível aplicar os conceitos financeiros revisados na seção 3. A análise econômica foi desenvolvida considerando as seguintes premissas: não há inflação energética, a geração de energia é constante durante todos os períodos do ano e não há queda de desempenho do sistema.

O primeiro passo para realizar a análise econômica consistiu em determinar o FC e o VPL, considerando uma vida útil de 25 anos para o sistema FV. A taxa mínima de atratividade utilizada foi a taxa SELIC (3\% a.a.). A SELIC foi escolhida por ser a taxa de juros de títulos públicos, ou seja, investimentos de baixo risco. Além do VPL, calculou-se o payback (em meses) e a TIR. O Quadro 1 mostra os indicadores financeiros VPL, payback e TIR obtidos.

Tabela 1 - Potência do gerador, quantidade de módulos, investimento, VPL, Payback e TIR.

\begin{tabular}{|c|c|c|c|c|c|c|c|}
\hline$\alpha$ & $\beta$ & $\mathrm{P}_{\text {gerador }}$ & $\mathrm{n}_{\text {modulos }}$ & $\mathrm{FC}_{0}$ & $\mathrm{VPL}$ & Payback & $\mathrm{TIR}$ \\
\hline $15 \%$ & $0 \%$ & $189 \mathrm{kWp}$ & 573 & $\mathrm{R} \$ 1.344 .246,0$ & $\mathrm{R} \$ 780.451,0$ & 131 & $8 \%$ \\
\hline $30 \%$ & $0 \%$ & $378 \mathrm{kWp}$ & 1146 & $\mathrm{R} \$ 1.870 .397$ & $\mathrm{R} \$ 2.355 .169,0$ & 91 & $12 \%$ \\
\hline $45 \%$ & $0 \%$ & $567 \mathrm{kWp}$ & 1718 & $\mathrm{R} \$ 2.396 .548$ & $\mathrm{R} \$ 3.929 .887,0$ & 78 & $15 \%$ \\
\hline $60 \%$ & $0 \%$ & $756 \mathrm{kWp}$ & 2291 & $\mathrm{R} \$ 2.922 .699,0$ & $\mathrm{R} \$ 5.504 .606,0$ & 72 & $17 \%$ \\
\hline $70 \%$ & $0 \%$ & $882 \mathrm{kWp}$ & 2672 & $\mathrm{R} \$ 3.273 .466,0$ & $\mathrm{R} \$ 6.554 .418,0$ & 69 & $17 \%$ \\
\hline $80 \%$ & $0 \%$ & $1008 \mathrm{kWp}$ & 3054 & $\mathrm{R} \$ 3.624 .234,0$ & $\mathrm{R} \$ 7.604 .230,0$ & 67 & $18 \%$ \\
\hline $90 \%$ & $0 \%$ & $1134 \mathrm{kWp}$ & 3436 & $\mathrm{R} \$ 3.975 .001,0$ & $\mathrm{R} \$ 8.654 .043,0$ & 65 & $18 \%$ \\
\hline $100 \%$ & $0 \%$ & $1260 \mathrm{kWp}$ & 3817 & $\mathrm{R} \$ 4.325 .768,0$ & $\mathrm{R} \$ 9.703 .855,0$ & 64 & $19 \%$ \\
\hline $50 \%$ & $50 \%$ & $684 \mathrm{kWp}$ & 2072 & $\mathrm{R} \$ 2.721 .640,0$ & $\mathrm{R} \$ 5.628 .251,0$ & 67 & $18 \%$ \\
\hline $100 \%$ & $100 \%$ & $1367 \mathrm{kWp}$ & 4143 & $\mathrm{R} \$ 4.625 .186,0$ & $\mathrm{R} \$ 12.050 .771,0$ & 57 & $21 \%$ \\
\hline
\end{tabular}

Analisando os dados da Tabela 1, verifica-se que o investimento é economicamente viável, pois em todos os casos o VPL foi maior que zero e a TIR foi maior que a TMA. Além do mais, nota-se que a sensibilidade dos indicadores econômicos TIR e payback, em relação ao investimento, diminui a partir do caso 4. Em geral, quanto maior for a potência instalada do sistema, maior será a viabilidade econômica do projeto.

\section{CONSIDERAÇÕES FINAIS}

Esse trabalho apresentou a utilização da metodologia PBL no curso de Engenharia Elétrica da Universidade Federal de Santa Maria campus Cachoeira do Sul com objeto de determinar a viabilidade econômica de um sistema fotovoltaico para um consumidor industrial do grupo A. Utilizando a PBL como metodologia de aprendizagem ativa, os discentes apresentaram retorno positivo, contribuindo em suas competências técnicas, sociais, empreendedoras e pessoais.

Como principais resultados, obteve-se uma aprendizagem ativa dos conteúdos utilizados para a resolução do problema, aperfeiçoando o trabalho em equipe, as habilidades técnicas, comunicação, liderança e, assim, contribuindo na formação profissional completa e autônoma desses alunos.

\section{REFERÊNCIAS}


ANEEL (2016). Micro e minigeração distribuída. Sistema de Compensação de Energia Elétrica. Brasília, DF, Brasil: Centro de Documentação-Cedoc.

BRASIL. Resolução $\mathrm{n}^{\circ}$ 2, de 24 de abril de 2019. Institui as Diretrizes Curriculares Nacionais do Curso de Graduação em Engenharia. Diário Oficial da União, Ministério da Educação/Conselho de Educação/Câmara de Educação Superior. Brasília, DF, 26 abr. de 2019. Ed. 80. Seção 1, p. 43.

FILHO, Gabriel Elmôr et al. Uma Nova Sala de Aula é Possível. $1^{\text {a }}$ ed. Rio de Janeiro: LTC, 2019.

MinhaCasaSolar. Suporte Fixação em Solo p/ 4 módulos de 230 a 350W RSO-232C. Disponível em: https://www.minhacasasolar.com.br/produto/suporte-fixacao-em-solo-p-4modulos-de-230-a-350w-rso-232c-79342. Acesso em: 23 mai. 2020.

NeoSolar. Painel Solar Fotovoltaico Canadian CSI CS6U-330P (330Wp). Disponível em: https://www.neosolar.com.br/loja/painel-solar/painel-solar-fotovoltaico-330wp-canadian-csics6u-330wp.html. Acesso em: 07 mai. 2020.

Pinho, J.T. and Galdino, M.A. (2014). Manual de engenharia para sistemas fotovoltaicos. Technical report, CEPEL-DTE-CRESESB.

Ross, S.A., Wester eld, R.W., Jordan, B.D., and Lamb, R. (2013). Fundamentos de administração financeira. AMGH Editora.

WINNERSHOP. Inversor Solar Fotovoltaico Sma Shp75-10 Sunny 75Kw Trifasico 380V Mppt Monitoramento. Disponível em: https://www.winnershop.com.br/inversorsolar/inversor-solar-fotovoltaico-sma-shp75-10-sunny-75kw-trifasico-380v-mpptmonitoramento/2907. Acesso em: 22 mai. 2020.

\section{METHODOLOGY OF ANALYSIS OF ECONOMIC VIABILITY FOR INSTALLING A PHOTOVOLTAIC SYSTEM THROUGH PROBLEM- BASED LEARNING}

Abstract: The Problem-Based Learning (PBL) process is an active learning method, where students learn certain subjects in the context of real problems. This article demonstrates the use of PBL in the Electrical Engineering course at the Federal University of Santa Maria campus Cachoeira do Sul. Thus, students are asked to analyze the economic viability of a photovoltaic system for an industrial consumer in group A. To solve the problem, a methodology for sizing, budgeting and economic analysis is used to install a photovoltaic system. The methodology was developed in an Excel spreadsheet to automate the calculations, which makes the model easily applicable to other case studies. As main results, it was obtained an active learning of the contents used to solve the problem, improving teamwork, technical skills, communication, leadership and, thus, contributing to the complete and autonomous professional formation of these students.

Keywords: active learning, problem-based learning, photovoltaic system, economic viability. 